\title{
Síndrome pulmonar e cardiovascular por hantavírus: aspectos clínicos de uma doença emergente no sudeste brasileiro
}

\author{
Pulmonary and cardiovascular syndrome due to hantavirus: \\ clinical aspects of an emerging disease in southeastern Brazil
}

\author{
Gelse Mazzoni Campos', Alessandra Abel Borges², Soraya Jabur Badra', \\ Glauciane Garcia Figueiredo ${ }^{1}$, Ricardo Luiz Moro de Souza ${ }^{3}$, Marcos Lázaro Moreli ${ }^{4}$ \\ e Luiz Tadeu Moraes Figueiredo ${ }^{1}$
}

\begin{abstract}
RESUMO
A síndrome pulmonar e cardiovascular por hantavírus é doença causada pela aspiração de aerossóis dos dejetos de roedores silvestres contaminados por vírus da família Bunyaviridae. Estudamos manifestações clínicas e laboratoriais de 70 casos ocorridos de 1998 a 2007 na região de Ribeirão Preto, SP. A frequiência de sintomas foi dispnéia (87\%), febre (81\%), tosse (44\%), cefaléia (34\%), taquicardia (81\%), hipotensão arterial (56\%), hipóxia (49\%), acidose metabólica (57\%), linfocitopenia (51\%), hematócrito >45\% (70\%), leucocitose com desvio à esquerda (67\%), creatinina (51\%) e uréia (42\%) séricas elevadas. A letalidade (54,3\%) ocorreu principalmente no $4^{\circ}$ dia. Insuficiência respiratória, hipotensão arterial e choque ocorreu 24-48 horas; o hematócrito elevado e a plaquetopenia são sinais fortemente sugestivos da doença. A hipótese diagnóstica de pneumonia atípica associada a bom prognóstico (p:0,0136); a infusão hídrica >2.000ml e hipotensão arterial associadas a mau prognóstico (p:0,0286 e p:0,0453).
\end{abstract}

Palavras-chaves: Hantavírus. Hantaviroses. Diagnóstico clínico. Epidemiologia. Doenças emergentes.

\begin{abstract}
Pulmonary and cardiovascular syndrome due to hantavirus is a disease caused by inhalation of aerosols from the excreta of wild rodents contaminated by viruses of the Bunyaviridae family. We studied the clinical and laboratory manifestations of 70 cases that occurred in the region of Ribeirão Preto, SP, Brazil, between 1998 and 2007. The frequency of symptoms was as follows: dyspnea (87\%), fever (81\%), coughing (44\%), headache (34\%), tachycardia (81\%), low arterial blood pressure (56\%), metabolic acidosis (57\%), lymphocytopenia (51\%), hematocrit > 45\% (70\%), leukocytosis with left deviation (67\%), creatinine (51\%) and urea (42\%). Mortality (54.3\%) occurred mainly on the fourth day. Respiratory insufficiency, low arterial blood pressure and shock occurred after 24 to 48 hours. High hematocrit and decreased platelet levels were signs strongly suggestive of the disease. The diagnostic hypothesis of atypical pneumonia was associated with a good prognosis $(\mathrm{p}=0.0136)$. Fluid infusion greater than $2,000 \mathrm{ml}$ and arterial hypotension were associated with a poor prognosis $(\mathrm{p}=0.0286$ and $\mathrm{p}=0.0453)$.
\end{abstract}

Key-words: Hantavirus. Hantavirosis. Clinical diagnosis. Epidemiology. Emerging diseases.

As doenças emergentes que são, em grande parte, frutos de alterações no ecossistema e dos comportamentos econômicos, sociais e culturais do homem, preocupam as autoridades sanitárias em todo o mundo. Estas doenças surgem como importante problema de saúde pública, tanto nas zonas rurais como nas urbanas. Dentre aquelas que despertam especial preocupação estão as febres hemorrágicas, como é o caso da síndrome pulmonar e cardiovascular por hantavírus (SPCVH),

\footnotetext{
1. Centro de Pesquisa em Virologia, Faculdade de Medicina de Ribeirão Preto Universidade de São Paulo, Ribeirão Preto, SP. 2. Unidade de Pesquisa em Virologia, Curso de Medicina da Universidade do Sul de Santa Catarina, Tubarão, SC. 3. Departamento de Ciências Básicas, Faculdade de Zootecnia e Engenharia de Alimentos, Universidade de São Paulo, Pirassununga, SP. 4. Departamento de Ciências Biológicas, Universidade Estadual Santa Cruz, Ilhéus, BA.

Address to: Dra Gelse Mazzoni. Campos Centro de Pesquisa em Virologia FMRP/USP. Av Bandeirantes 3900, 14049900 Ribeirão Preto, SP

Telefax: 5516 3602-4508/3620-9726

e-mail: gelse@usp.br

Recebido para publicação em 24/09/2008

Aceito em 03/04/2009
}

devido ao seu caráter comumente letal e à sua ocorrência em praticamente todo o continente americano. Esta doença foi descrita pela primeira vez em 1993, quando ocorreram mortes repentinas por grave pneumonia, na região de Four Corners, no sudoeste dos Estados Unidos.

Os hantavírus são esféricos, RNA de fita simples, pertencem ao gênero Hantavirus da família Bunyaviridae. A transmissão destes vírus se processa pela aspiração de excretas contaminadas de roedores silvestres; em nossa região representados pelo rato do rabo peludo (Necromys lasiurus), rato da mata (Akodon $s p$ ) e ratinho do arroz (Oligoryzomys $s p)^{7}$. É favorecida pela proximidade dos seres humanos com fontes contaminadas na zona rural, seja em atividades de trabalho ou lazer, sendo os adultos da área rural com mais fatores de risco para a infecçã $0^{21}$. 0 período de incubação descrito de 9 a 33 dias, com média de 14 a 17 dias $^{30}$.

Nas primeiras descrições desta síndrome, os doentes apresentavam um quadro influenza-símile, com febre, cefaléia, mialgias e calafrios, que progredia para insuficiência respiratória 
grave. Em quase 50\% dos casos, ocorria choque cárdiocirculatório; daí porque a doença passou a ser denominada síndrome pulmonar e cardiovascular por hantavírus ${ }^{12} 1325$.

A contagem de plaquetas abaixo de $130.000 / \mathrm{mm}^{3}$; hemoconcentração maior que 55\%, encontrada em $78,6 \%$ dos casos; leucocitose com níveis acima de $15.000 / \mathrm{mm}^{3}$, neutrofilia maior que $7.000 / \mathrm{mm}^{3}$ e formas jovens de bastonetes acima de $600 / \mathrm{mm}^{3}$, em $64,2 \%$ dos casos. Creatinina sérica acima de $1,5 \mathrm{mg} / \mathrm{dl}$ foi observada em $63,6 \%$ dos casos e hipoxemia com $\mathrm{PO}_{2}$ inferior a $70 \mathrm{mmHg}$ em $54,5 \%$.

Os achados radiológicos pulmonares da SPCVH descritos como semelhantes aos encontrados em outras viroses, como influenzas, sarampo, adenoviroses, herpesviroses, varicella-zoster e citomegaloviroses ${ }^{14}$. No período inicial, apresenta opacidade peri-hilar e basal e acentuação lateral das fissuras. Após 4 dias, observa-se infiltrado alveolar bilateral difuso até a porção média dos pulmões, com inversão da trama bronquioalveolar.

Na fase inicial, os sintomas são semelhantes à influenza tipos A e B, resfriado comum, gastroenterite inespecífica ou mesmo dengue. Na segunda fase, pode ser confundida com estafilococcias, pneumonia atípica por Chlamydia, Parainfluenza tipo 4, Paramyxovírus, ou Mycoplasma, Leptospirose sp (forma pulmonar), pneumocistose e septicemia, além de outras causas não pulmonares, como valvulopatias e síndrome de Goodpasture ${ }^{23}$.

0 diagnóstico laboratorial da infecção aguda por hantavírus pode ser feito por método sorológico, sendo muito utilizado no Brasil o ELISA com detecção de IgM e IgG contra antígenos de vírus Sin Nombre ou Los Andes ${ }^{8}$ e por RT-PCR, amplificando os genes das proteínas $\mathrm{N}$ e G1, diretamente do sangue ou fragmento de órgão dos pacientes ${ }^{20}$. Nos casos fatais, a reação de imunohistoquímica em tecidos costuma ser utilizada para confirmar o diagnóstico. Neste último teste, utiliza-se o anticorpo monoclonal de camundongo anti-hantavírus Puumala (1:200, clone GB04-BF07, CDC - Atlanta, EUA) ${ }^{25}$.

No tratamento da SPCVH, recomendam-se, em qualquer caso, medidas imediatas de suporte respiratório, que em casos gravíssimos utiliza a oxigenação extra corpórea ${ }^{10}$, restrição hídrica, controle do choque com aminas vasoativas e monitoramento do paciente em terapia intensiva ${ }^{8}$. Estudos realizados na China com pacientes com FHSR sugerem que a aplicação de ribavirina endovenosa, por 7 dias, produz uma melhora no prognóstico quando utilizada precocemente no curso da doença, diminuindo a morbidade e mortalidade ${ }^{24}$.

A prevenção das infecções por hantavírus baseia-se em medidas educativas tomadas junto às comunidades, direta ou indiretamente relacionadas com o meio rural, no sentido de prevenir o contato com os roedores ou suas excretas.

OBrasil diagnostica casos de hantavirose desde 1993 e, desde então, até julho de 2008, foram notificados cerca de 1.000 casos de SPCVH. Os casos diagnosticados em território brasileiro apresentam diferenças sintomáticas variáveis de acordo com a região de ocorrência.

Sendo a SPCVH uma doença de descrição recente e com características emergentes, é importante que seja minuciosamente estudada, utilizando metodologias variadas para identificar particularidades epidemiológicas, bem como, diferentes manifestações clínicas e laboratoriais nos pacientes infectados por hantavírus, visando a obter um melhor conhecimento do comportamento da doença no país, tão necessário para planejar estratégias de tratamento e controle da doença.

\section{MATERIAL E MÉTODOS}

Este estudo, realizado durante o período de 1998 a 2007, foi aprovado pelo Comitê de Ética em Pesquisa, do Hospital das Clínicas da Faculdade de Medicina de Ribeirão Preto, da Universidade de São Paulo (processo número 2657/03). Dos pacientes com SPCVH, coletamos informações sobre sintomas e sinais apresentados, valores laboratoriais observados e tratamento ministrado, preenchendo Ficha Padrão de Avaliação Clínica, de prontuários hospitalares e fichas da Divisão de Vigilância Epidemiológica da Secretaria Municipal de Saúde e Departamento Regional de Saúde de Ribeirão Preto (DRS-XIII). A análise estatística dos dados foi realizada com o programa InStat 4.0 (GraphPad Software, Inc., EUA) pelo teste exato de Fischer com nível de significância de 5\%.

\section{RESULTADOS}

Analisamos retrospectivamente aspectos epidemiológicos, clínicos, laboratoriais e o tratamento de 70 casos de SPCVH com diagnóstico laboratorial no Centro de Pesquisa em Virologia da Faculdade de Medicina de Ribeirão Preto, Universidade de São Paulo. Destes casos, 53 (75,7\%) eram do sexo masculino, sendo $48(68,5 \%)$ destes tinham entre 20 e 40 anos, idade média de $35,8 \pm 11,7$ anos; e os 17 (24,3\%) participantes do sexo feminino tinham idade média de 28,8 $\pm 11,9$ anos.

Dos casos estudados, 15 (22\%) pacientes informaram com razoável segurança a data provável de infecção. Em 12 (80\%) destes casos, $o$ tempo entre o provável contato com 0 vírus e 0 aparecimento dos sintomas ficou compreendido entre 2 e 7 dias (média de 4,7 dias) e nos 3 (20\%) casos restantes, o período de incubação foi de 30 dias.

Foi possível obter a data de início dos sintomas (DIS) em relação a 43(61,4\%) pacientes. A data da internação hospitalar (DIH) ocorreu, na maioria dos casos (66\%), entre o terceiro e o sexto dia após a DIS. A data de ocorrência do óbito (DOO), na maioria (42\%) dos pacientes ocorreu no quarto dia após a DIS.

Os sintomas prodrômicos, apresentados desde a DIS até a DIH, muito inespecíficos são mostrados na Tabela 1; exceto duas pacientes que apresentaram otalgia e tiveram diagnóstico inicial de otite média aguda.

Os sintomas mais comuns entre os pacientes com SPCVH ocorreram da seguinte forma: febre em 35 (81\%) pacientes perdurou até o quinto dia de doença $\mathrm{D} 5$; a tosse iniciou-se na DIS em $50 \%$ dos casos e o restante entre o D2 o D5; a dispnéia leve manifestou-se entre o D2 e o D6, nas formas moderadas entre D3 a D5 e D6 nas graves; a cianose, observada em 8 (18\%) pacientes ocorreu em D5 e D6; a adinamia, em 20 (47\%) pacientes, a mialgia e a cefaléia, descritas na maioria dos casos, ocorreram de D1 a D4, com localização retro-orbitária. Os sintomas 
TABELA 1

Sintomas prodrômicos síndrome pulmonar e cardiovascular por hantavírus.

\begin{tabular}{lrc}
\hline Sinais e sintomas & $\begin{array}{r}\text { Casos } \\
\left(\mathrm{n}^{\mathrm{o}}\right)\end{array}$ & $\begin{array}{c}\text { Freqüência } \\
(\%)\end{array}$ \\
\hline Febre & 35 & 81,0 \\
Adinamia & 20 & 47,0 \\
Tosse & 19 & 44,0 \\
Dispnéia & 15 & 34,0 \\
Cefaléia & 15 & 34,0 \\
Náuseas e vômitos & 11 & 25,0 \\
Indisposição & 9 & 21,0 \\
Mialgia & 8 & 18,0 \\
Diarréia & 6 & 14,0 \\
Fenômenos hemorrágicos & 4 & 9,0 \\
Gripe forte & 2 & 4,0 \\
Otalgia & 2 & 4,0 \\
Hipotensão & 1 & 2,0 \\
Choque & 1 & 2,0 \\
\hline
\end{tabular}

gastrointestinais observados em 11 (25\%) casos incluíram náuseas e vômitos, entre D1 e D5 e também, diarréia em 6 casos na metade dos casos surgindo na DIS e nos demais de D3 a D5. Fenômenos hemorrágicos, como hematúria, hematêmese, melena e metrorragia, ocorreram, na maioria dos casos, de D3 a D5; hipotensão arterial, observada em 19 (44\%) pacientes, ocorreu de D4 a D7. 0 choque, observado em 14 (33\%) casos, ocorreu de D4 a D6. Outros sintomas, como convulsões, síncopes, vertigem e tonturas, hemiplegia, dor abdominal e lombar, anúria, edema de pés ou face e otalgia, ocorreram em 23 (53\%) pacientes.

Dados referentes ao exame físico realizado no momento da internação hospitalar foram obtidos de $43(61,4 \%)$ dos pacientes com SPCVH. Este exame evidenciou mau estado geral em 18 (42\%) pacientes, taquidispnéia (frequência respiratória -fr maior que $22 \mathrm{ipm}$ ) em 38 (88\%) pacientes, em 17 (40\%) desses casos, a frequiência respiratória superou $32 \mathrm{ipm}$. Temperatura corpórea superior a $38^{\circ} \mathrm{C}$ foi observada em 33 (77\%) casos, instabilidade hemodinâmica, com frequiência cardíaca maior que 100 bpm, em 35 (81\%) casos e hipotensão arterial em 24 (56\%) pacientes. 0 choque foi relatado em 5 (10\%) casos e cianose em 11 (26\%). Três casos tiveram nível de consciência alterado com o teste de Glasgow de 3 a 5, p ara a maioria em torno de 15 .

No momento da internação em $43(61,4 \%)$ pacientes com SPCVH cujos dados hematimétricos foram disponibilizados, 30 (70\%) apresentaram hematócrito maior que 45\% em sendo, e 23 (54\%), acima de 50\%. As taxas de hemoglobina mostraramse elevadas em 26 (60\%), normais em 12 (28\%) e diminuídas (anemia hipocrômica) em 5 (12\%) pacientes. Nestes 5 últimos casos, o hematócrito apresentou valores normais ou diminuídos. Com relação ao leucograma, 6 (14\%) pacientes apresentaram leucopenia com contagem de glóbulos brancos $<5.000$ células/ $\mathrm{mm}^{3} ; 27$ (63\%) exibiram leucocitose (média de 14.556 células $/ \mathrm{mm}^{3}$ ) e nestes o desvio à esquerda apresentou-se em 29 (67\%) casos, inclusive com presença de metamielócitos em 11 (26\%) casos. Linfocitopenia (em média $765 \pm 235$ células por $\mathrm{mm}^{3}$ ) foi detectada em 18 (51\%) casos. Os teores médios de plaquetas foram de $94.110 / \mathrm{mm}^{3}$, sendo que 40 (93\%) pacientes apresentaram valores inferiores a 150.000 plaquetas $/ \mathrm{mm}^{3} \mathrm{e}$ destes, 32 (74\%) tiveram menos que 100.000 plaquetas $/ \mathrm{mm}^{3}$.

Nos testes de função renal, os teores de uréia $(45,26$ $\pm 26,93 \mathrm{mg} / \mathrm{dl}$ ) revelaram-se aumentados em 18 (42\%) pacientes e os de creatinina $(1,38 \pm 0,59 \mathrm{mg} / \mathrm{dl})$ em $22(51 \%)$. Quanto às dosagens enzimáticas, os teores de TGO/AST $(90,76 \pm 65,74 \mathrm{U} / \mathrm{l})$ e TGP/ALT $(83,18 \pm 70,67 \mathrm{U} / 1)$ apresentaram-se elevados em 31 (72\%) e $36(84 \%)$ pacientes, respectivamente. Nos 6 pacientes que realizaram o exame, a DHL e a creatino fosfoquinase (CK) mostraram-se elevadas.

Quanto ao exame gasométrico, realizado em 35 pacientes, 20 (57\%) apresentaram sangue arterial com pH ácido e 15 (43\%) tinham $\mathrm{pH}$ na faixa normal. Porém, em relação ao base excess (BE), 28 (80\%) pacientes apresentaram acidose e para apenas 7 (20\%) os resultados foram normais. Quanto à saturação de hemoglobina pelo $0_{2}, 21(60 \%)$ dos pacientes apresentaram hipóxia, com níveis de saturação de oxigenação na hemoglobina $<90 \%$ e o restante, níveis normais de saturação.

Em síntese, as principais manifestações clínicas da SPCVH foram: dispnéia em 38 (87\%) pacientes, febre em 35 (81\%), tosse em 19 (44\%) e cefaléia em 15 (34\%). Ao exame físico, foram taquicardia em 35 (81\%) pacientes e hipotensão arterial em 24 (56\%). À oximetria de pulso, observou-se diminuição da saturação de hemoglobina por $\mathrm{O}_{2}$ em 21 (49\%) pacientes. As principais alterações bioquímicas e hematimétricas foram, plaquetopenia $\left(<150.000\right.$ plaquetas por $\left.\mathrm{mm}^{3}\right)$ em $40(93 \%)$ pacientes, acidose em 20 (57\%), linfocitopenia em 18 (51\%), hematócrito maior que $45 \%$ em 30 (70\%) pacientes, leucocitose com desvio à esquerda em 29 (67\%), uréia elevada em 18 (42\%) e aumento de creatinina em 22 (51\%). Estes dados são mostrados na Tabela 2.

Colocando estes dados segundo uma visão evolutiva dos casos com SPCVH, observou-se febre, tosse seca, náuseas e vômitos de entre os dias 1 e 5 após o aparecimento dos sintomas (D1 a

TABELA 2

Clínicas e laboratório na síndrome pulmonar e cardiovascular por hantavirus.

\begin{tabular}{lcc}
\hline Sinais e sintomas* & Casos** & Frequência \\
& & $\%$ \\
\hline Plaquetopenia $<150.000 / \mathrm{mm}^{3}$ & 40 & 93,0 \\
Dispnéia & 38 & 87,0 \\
Taquicardia & 35 & 81,0 \\
Febre & 35 & 81,0 \\
Linfocitopenia**** & 18 & 51,0 \\
Hematócrito >45\% & 30 & 70,0 \\
Leucocitose c/ desvio à esquerda & 29 & 67,0 \\
Hipotensão & 24 & 56,0 \\
Creatinina elevada & 22 & 51,0 \\
Saturação hemoglobina $0_{2}<90$ & 21 & 49,0 \\
Acidose & 20 & 57,0 \\
Tosse & 19 & 44,0 \\
Uréia elevada & 18 & 42,0 \\
Cefaléia & 15 & 34,0 \\
\hline
\end{tabular}

*Em ordem decrescente de frequiência, ***Para um total de $n^{0}=43$ casos, ****ara um total de $n^{0}=35$ casos 
D5); adinamia, mialgia e cefaléia foram observados de D1 a D4 e diarréia de D2 a D5. Após três dias do aparecimento dos sintomas surgem a doença respiratória cursando com tosse seca e dispnéia, inicialmente leve, mas que evolui rapidamente para dispnéia grave em 24 a 48 horas. Cianose ocorreu de D3 a D6. Verificaram-se fenômenos hemorrágicos de D3 a D5, e hipotensão arterial e choque de D4 a D7. Estes dados mostrados na Figura 1.

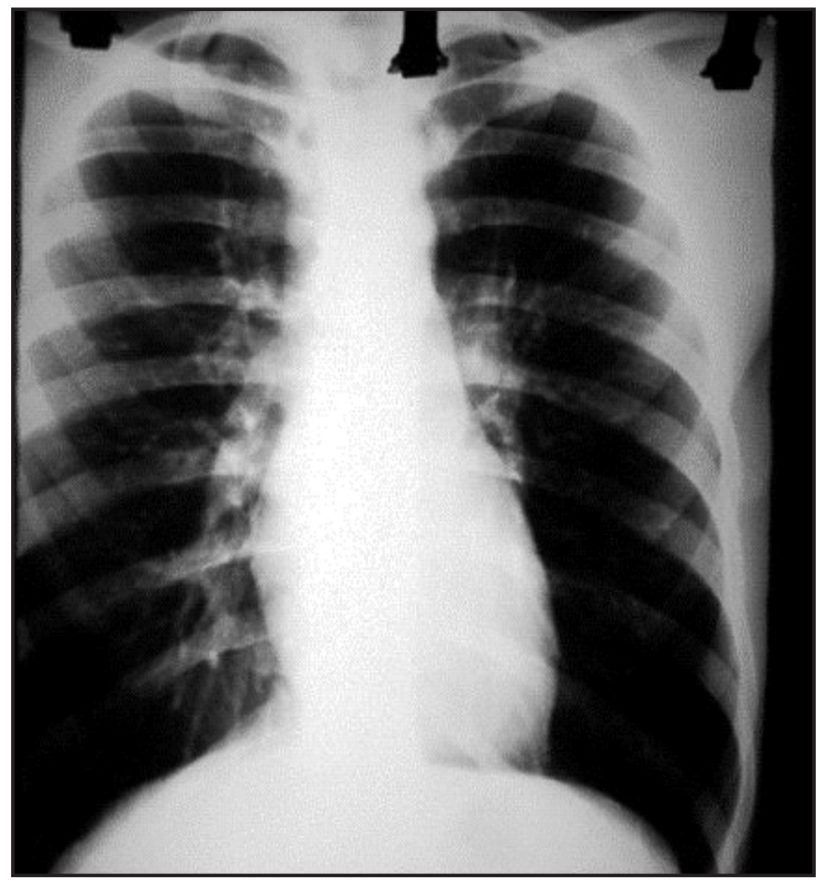

FIGURA 1

Evolução clínica dos casos de síndrome pulmonar e cardiovascular por hantavirus.

Com base em 29 pacientes com SPCVH, dos quais se teve acesso a exames radiológicos obtidos em diferentes fases da doença, observou-se acometimento pulmonar bilateral do tipo intersticial difuso em 12 (41\%) casos, como na Figura 2; do tipo alveolar difuso bilateral em 13 (41\%) casos, sendo que 4 exibiam padrão compatível com SARA, como na Figura 3; 4 (14\%) casos exibiram padrão retículo-nodular e/ou flocoso e 3 pacientes apresentaram derrame pleural.

Quanto ao diagnóstico laboratorial de infecção por hantavírus, dos 70 pacientes estudados, 64 (91\%) tiveram confirmação sorológica por ELISA pesquisando anticorpos IgM específicos em testes realizados pelo Instituto Adolfo Lutz de São Paulo (SP). Para 34 (49\%) destes casos a confirmação diagnóstica foi feita por RT-PCR e ELISA no CPV- FMRP-USP. Seis (9\%) casos fatais tiveram diagnóstico confirmado por imunohistoquímica, realizada pelo Instituto Adolfo Lutz.

Quanto à evolução dos pacientes, durante a internação, todos os 38 (54,3\%) óbitos por SPCVH ocorreram em 1,3 \pm 0,5 dias após a admissão. Os 32 sobreviventes tiveram tempo médio na internação hospitalar de 8,9 \pm 4,5 dias.

Informações relativas ao tratamento a que foram submetidos os pacientes com SPCVH são mostradas na Tabela 3. Com base em 31 (44,3\%) pacientes, dos quais se obteve informação sobre

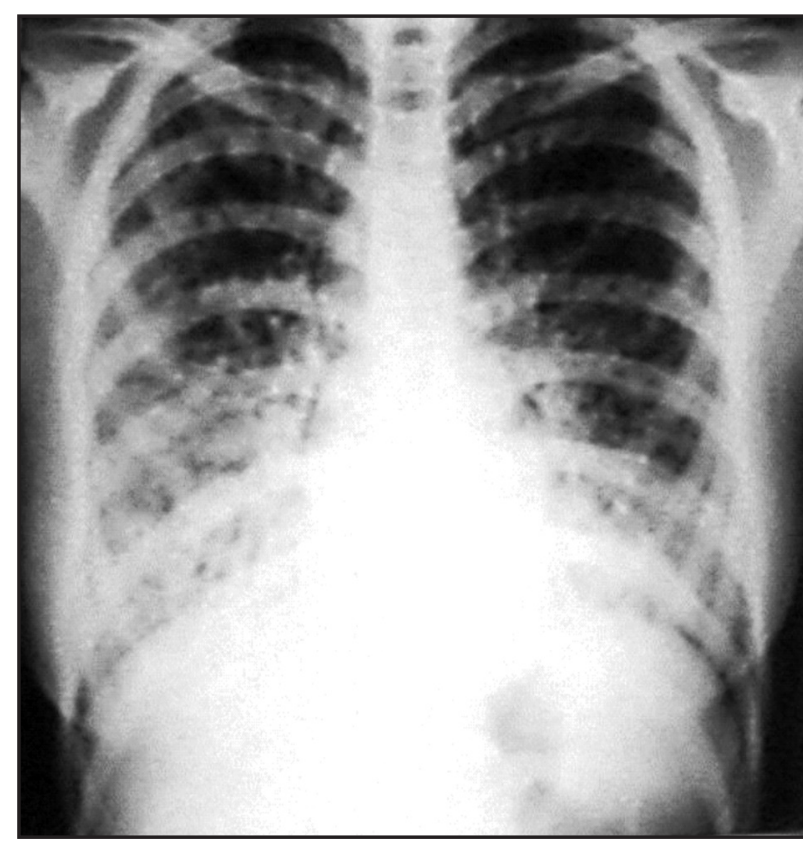

FIGURA 2

Radiografia de tórax de paciente em D2 da síndrome pulmonar e cardiovascular por hantavírus.

\begin{tabular}{|l|l|l|l|l|l|l|l|l||}
\hline Dias de Evolução* & D1 & D2 & D3 & D4 & D5 & D6 & D7 & D8 \\
\hline Febre & & & & & & & & \\
\hline Tosse & & & & & & & & \\
\hline Vômitos & & & & & & & & \\
\hline Náuseas & & & & & & & & \\
\hline Adinamia & & & & & & & & \\
\hline Mialgia & & & & & & & & \\
\hline Cefaléia & & & & & & & & \\
\hline Diarréia & & & & & & & & \\
\hline Dispnéia & & & & & & & & \\
\hline Cianose & & & & & & & \\
\hline Fenom.Hemorria & & & & & & & \\
\hline Hipotensão/choque & & & & & & & & \\
\hline
\end{tabular}

FIGURA 3

Radiografia de tórax de paciente em D4 da síndrome pulmonar e cardiovascular por hantavírus.

volumes de hidratação, observou-se que $64 \%$ dos que receberam infusão hídrica $>2.000 \mathrm{ml}$ nas primeiras $24 \mathrm{~h}$ de internação foram a óbito, enquanto que sobreviveram $78 \%$ dos que receberam infusão hídrica $<2.000 \mathrm{ml}$ (p:0,0286).

De $41(58,8 \%)$ pacientes sobre os quais há relato de terem sido submetidos a suporte respiratório, 19 (46\%) receberam oxigenioterapia (máscara facial e cateter nasal de $\mathrm{O}_{2}$ ) e 22 (54\%) receberam ventilação mecânica; destes últimos, 15 foram a óbito e 7 sobreviveram. Ressalta-se que todos os pacientes que faleceram foram submetidos à ventilação mecânica, exceto os que já chegaram em óbito, o qual foi constatado no hospital. Observou-se correlação entre suporte respiratório invasivo e má evolução da doença (p:0,0011). 
TABELA 3

Tratamento da síndrome pulmonar e cardiovascular por hantavírus.

\begin{tabular}{|c|c|c|c|c|c|c|c|}
\hline \multirow[b]{3}{*}{ Suporte terapêutico } & & & \multicolumn{4}{|c|}{ Freqüência } & \multirow[b]{3}{*}{$\mathrm{P}$} \\
\hline & \multicolumn{2}{|c|}{ Casos } & \multicolumn{2}{|c|}{ cura } & \multicolumn{2}{|c|}{ óbito } & \\
\hline & $\mathrm{n}^{\circ}$ & $\%$ & $\mathrm{n}^{\circ}$ & $\%$ & $\mathrm{n}^{\circ}$ & $\%$ & \\
\hline Hidratação & 31 & 100,0 & 18 & 100,0 & 13 & 100,0 & 0,0286 \\
\hline infusão hídrica $>2.000 \mathrm{ml}$ & 12 & 39,0 & 5 & 42,0 & 7 & 58,0 & \\
\hline infusão hídrica $<2.000 \mathrm{ml}$ & 19 & 62,0 & 13 & 78,0 & 6 & 22,0 & \\
\hline Suporte respiratório & 41 & 100,0 & 25 & 100,0 & 16 & 100,0 & 0,0011 \\
\hline ventilação mecânica & 22 & 54,0 & 7 & 28,0 & 15 & 94,0 & \\
\hline oxigenioterapia & 19 & 46,0 & 18 & 72,0 & 1 & 6,0 & \\
\hline Corticóides & 46 & 100,0 & 25 & 100,0 & 21 & 100,0 & 0,7624 \\
\hline $\operatorname{sim}$ & 17 & 36,0 & 10 & 40,0 & 7 & 34,0 & \\
\hline não & 29 & 64,0 & 15 & 60,0 & 14 & 66,0 & \\
\hline Aminas vasoativas & 45 & 100,0 & 24 & 100,0 & 21 & 100,0 & 0,0345 \\
\hline $\operatorname{sim}$ & 16 & 36,0 & 5 & 21,0 & 11 & 53,0 & \\
\hline não & 29 & 64,0 & 19 & 79,0 & 10 & 47,0 & \\
\hline
\end{tabular}

Quanto ao uso de corticóides, de $46(65,7 \%)$ pacientes dos quais foi possível obter este dado, 17 (37\%) foram medicados com estas drogas e destes, 7 (15\%) foram a óbito. Dos 29 (63\%) não tratados com corticóides, 14 (30\%) foram a óbito. Portanto, a letalidade no grupo tratado com corticóides foi de $41 \%$, contra $48 \%$ no grupo não tratado, diferença não significante (p:0,7624). Quanto ao uso de aminas vasoativas, em 45 (64,3\%) pacientes dos quais foi possível obter este dado, verificou-se que elas foram utilizadas no tratamento de 16 (36\%). Neste grupo, verificou-se letalidade de $53 \%$, contra $47 \%$ no grupo não tratado com estes medicamentos, diferença não significante (p:0,0345).

\section{DISCUSSÃO}

Na região de Ribeirão Preto e outros locais de origem dos pacientes aqui estudados (região de campo cerrado e planalto central) o causador da SPCVH é o vírus Araraquara (ARAV). Este vírus possui como reservatório natural o roedor silvestre Necromys lasiurus que sabidamente produz os quadros mais graves e com maior letalidade de SPCVH, dentre todos os hantavírus brasileiros. 0 presente trabalho descreve a SPCVH por ARAV ${ }^{620}$.

0 perfil dos 70 casos de SPCVH, analisados neste estudo, revelaram diferentes dados de procedência, gênero e faixa etária dos indivíduos. Em geral adultos jovens e de meia idade exercendo atividades profissionais diversas. Observamos neste estudo, em contraposição dos dados da literatura que a maioria dos casos com período de incubação curto, de 2 a 7 dias, sendo menos frequientes as ocorrências com 7 a 30 dias. Em estudo recente realizado no Chile, investigando a transmissão interpessoal de Hantavírus no ambiente doméstico, 16 pessoas contactantes e que desenvolveram a SPCVH tiveram um período de incubação de 16 a 23 dias 5. Outros estudos, realizados nos Estados Unidos, Chile, Argentina e Brasil referem período de incubação entre 9 e 39 dias, com média de 18 dias $^{5630}$. Sabendo que distintos hantavírus americanos produzem SPCVH com diferentes manifestações clínicas e com níveis diversos de gravidade, atribuímos esta divergência de dados, provavelmente, à maior virulência do hantavírus Araraquara que ocorre na região $0^{18}$.

Quanto à SPCVH, observamos que febre e mialgia ocorrem desde os pródromos da doença. Entretanto, por serem sinal e sintoma inespecíficos, não auxiliam no diagnóstico diferencial em fase inicial da doença; em concordância com outros autores que referem os pródromos da SPCVH como inespecíficos ${ }^{2627}$. Concordamos com outros autores quanto ao período de 4 a 5 dias entre DIS e DIH e ainda, quanto à DOO de 2,5 a 5,5 dias ${ }^{1522} 28$.

A frequiência dos sintomas prodrômicos tem sido variável em diferentes estudos sobre SPCVH. Porém, alguns estão presentes e com alta frequiência em todos, como febre, mialgia e tosse, em 100\% dos casos de Mato Grosso e $65 \%$ na casuística paranaense ${ }^{1922}$. Entretanto, no estudo matogrossense e em nossa casuística, a presença de mialgia e tosse é de frequiência menor, atingindo apenas a metade dos casos ${ }^{19}$.

A dor torácica sem falta de ar, pouco referida em nossa casuística, esteve presente com frequiência em torno de $60 \%$ em outros estudos ${ }^{1622} 26$. A dor abdominal, com ou sem diarréia, foi relatada por outros autores em 20 a 30\% de $\operatorname{casos}^{226}$. Enquanto neste trabalho, foi observado diarréia sem dor abdominal em $14 \%$ de casos. Por outro lado, encontramos otite média em dois casos, não encontrada em outros estudos, o que pode sugerir uma diversidade sintomática da doença.

A evolução da insuficiência respiratória na SPCVH caracterizou-se pouca intensidade no início (D3 ou D4), piorando rapidamente (D4 ou D5) e tornando-se grave em 24 a 48 horas (D4 a D6). Descrição de uma evolução semelhante tem sido mostrada na maioria dos estudos realizados sobre a doença nas Américas ${ }^{913}$. A insuficiência respiratória e sua progressão característica sugerem o diagnóstico de SPCVH para o médico assistente. Entretanto o diagnóstico quando feito em paciente com grave insuficiência respiratória não é tão útil ao paciente quanto o realizado precocemente.

Observamos nos pacientes estudados, casos de diarréia como sintoma proeminente, que em 30 a 50\% dos casos ocorreu no 
início da doença (D3), geralmente acompanhada por náuseas e vômitos, com duração de 2 a 3 dias. Este acometimento digestivo representa mais um fator complicador ao diagnóstico de SPCVH, pois confunde o pensamento clínico direcionando-o para 0 diagnóstico de gastroenterocolite aguda. Especialmente se o quadro se fizer acompanhar de hipotensão arterial, tende-se a utilizar hidratação vigorosa, que de acordo com nossos resultados, tende a agravar a SPCVH.

Fenômenos hemorrágicos foram relatados em casos brasileiros de SPCVH com frequiências semelhantes à do presente estudo, em torno de 10 a $20 \%{ }^{216}$ e associados ao quadro febril, reportam a diagnósticos diferenciais com dengue hemorrágica.

Neste estudo duas pacientes que foram a óbito apresentaram sintomas neurológicos com sinais de localização, cuja suspeita à internação foi de acidente vascular encefálico. Nestes casos a necropsia revelou hemorragia em núcleos da base, provavelmente fenômeno hemorrágico conseqüente à alteração vascular e plaquetopenia. Quadros semelhantes acrescidos de convulsões, síncopes, vertigem, tonturas e hemiplegia foram encontrados por outros autores ${ }^{45}$. Estas manifestações clínicas pouco comuns não levam à suspeita de hantavirose, inviabilizando um diagnóstico precoce de SPCVH.

Dores abdominais e lombares, anúria e edema nos membros e face, foram manifestados por alguns pacientes de forma isolada, podendo estar relacionados a acometimento renal, com elevação nos níveis de uréia e creatinina, o qual é encontrado em 18 a $60 \%$ dos casos por vários autores ${ }^{11} 22272831$. Alguns citam a síndrome nefrótica em $8 \%$ dos casos ${ }^{4}$.

Os pacientes com SPCVH, quando atendidos já de início em estado grave, apresentavam febre e mau estado geral, com ou sem insuficiência respiratória (frequiência respiratória >22ipm), taquicardia e hipotensão arterial. Nestes casos a instabilidade hemodinâmica piorava rapidamente evoluindo para choque, podendo levar o paciente à morte na dependência de uma intervenção terapêutica adequada e imediata. Entretanto relatamse casos moderados de SPCVH que rapidamente evoluem para formas muito graves ${ }^{528}$.

A instabilidade hemodinâmica mostrou-se parâmetro indicador de gravidade na SPCVH. A taquicardia presente em 80\% dos casos e que aparece entre D3 e D6 é o primeiro sinal de instabilidade hemodinâmica que alerta para a SPCVH muito grave. A hipotensão arterial, presente em mais de $50 \%$ dos casos, é um sinal reversível quando precocemente tratada, prevenindo a ocorrência do choque ${ }^{1622}$. A cianose e a diminuição da saturação de hemoglobina por $\mathrm{O}_{2}$, no oxímetro de pulso, revelando 0 grau de hipóxia do paciente e, quando presentes, indicaram a gravidade do caso.

A leucocitose com acentuado desvio à esquerda, por nós observada, tem sido descrita pela maioria dos autores que estudam a doença $a^{2} 62627$. Quanto a achados laboratoriais, segundo Saggioro ${ }^{25}$, a linfocitose tem correlação com a patogênese da SPCVH, fazendo da linfocitopenia, observada na maioria dos casos, fato inusitado, que pode sugerir demanda linfocitária não acompanhada por produção medular.
A elevação do hematócrito é descrita como um dos sinais mais característicos da SPCVH porque reflete a hemoconcentração conseqüente à síndrome de extravasamento capilar em leito vascular pulmonar própria da doença. Observamos alta frequiência de hematócrito elevado, 70 a $80 \%$ dos casos, o que foi condizente com o descrito por outros autores ${ }^{1622}$ os quais chegam a descrever esta alteração em até $100 \%$ de suas casuísticas². Em alguns casos correlacionamos a não elevação do hematócrito a doenças prévias, como a anemia.

A plaquetopenia na SPCVH representa consenso, assim como em nossa casuística, é citada pela maioria dos autores ${ }^{7} 81013$. Ela, juntamente com a elevação do hematócrito é considerada referencial para a suspeita de SPCVH.

Quanto à função renal, teores elevados de uréia e creatinina foram mais encontrados entre os sobreviventes da SPCVH, o que nos faz supor que o quadro de insuficiência renal se desenvolve após o quarto dia de doença, ocasião, também, em que ocorreu o maior número de óbitos.

As dosagens de enzimas hepáticas mostraram-se elevadas em 83\% dos pacientes com SPCVH, sugerindo comprometimento de hepatócitos. Entretanto não se observou hepatomegalia nestes pacientes. Observou-se elevação da CK sérica, o que poderia estar relacionado ao esforço da musculatura respiratória pela dispnéia ou associada à infecção do tecido muscular pelo Hantavírus.

A acidose metabólica esteve presente em $81 \%$ dos pacientes submetidos à gasometria arterial e mostrou-se, como veremos adiante, um fator determinantes de mau prognóstico nos casos de SPCVH.

Analisando as hipóteses diagnósticas formuladas pelos médicos que atenderam inicialmente os pacientes com SPCVH, observou-se que pneumonia atípica se associou à sobrevivência do paciente (p:0,0327). As hipóteses de insuficiência respiratória aguda e Hantavirose tenderam a ser mais observadas em sobreviventes. Outros autores têm observado a associação entre severidade, suspeita diagnóstica e tratamento de casos de SPCVH em unidades de terapia intensiva ${ }^{25}$.

Quanto aos exames radiológicos de tórax dos pacientes, com SPCVH, observa-se um padrão de acometimento bilateral intersticial e difuso no início do quadro (entre D1 e D4) e com o agravamento da insuficiência respiratória, o quadro evolui com infiltrado alveolar, com ou sem inversão da trama brônquico-vascular, traduzindo condensação pulmonar de natureza inflamatória ou congestiva. Dois pacientes apresentaram derrame pleural bilateral após D4, denotando piora do quadro congestivo. Com base no quadro radiológico, outros autores chamam atenção para um diagnóstico diferencial com outras causas de Pneumonia Atípica em pacientes imunocompetentes, como aquela pelos vírus Influenza tipos A e B. Quanto a pacientes imunocomprometidos, o quadro radiológico de SPCVH poderia ser confundido com pneumonias por fungos como o Pneumocistis carinii e o bistoplasma, além daquelas de etiologia viral, por citomegalovírus, herpesvírus, vírus do sarampo e adenovírus ${ }^{14}$.

Buscando por uma associação entre sinais e sintomas apresentados pelos pacientes com SPCVH e a evolução do quadro, 
observaram-se praticamente as mesmas entre os casos fatais e os sobreviventes, diferenciando-se apenas pela gravidade. Observouse que o quarto dia após o surgimento dos sintomas (D4) é um dia crítico na evolução da SPCVH, pois foi o de maior ocorrência para internação hospitalar (DIT) e para o óbito (DOT). Neste dia, o diagnóstico pode ser suspeitado pela manifestação de insuficiência respiratória grave em paciente previamente hígido e o tratamento adequado pode ser instituído; a partir daí, o quadro evolui para a cura ou, quando não, o doente piora rapidamente e morre, quase sempre no mesmo D4.

A acidose metabólica detectada pela gasometria arterial nos pacientes suspeitos de SPCVH deve ser diagnosticada e tratada, pois observamos associação com mau prognóstico. Dados são semelhantes aos encontrados na literatura, em que falência respiratória e choque são associados a mau prognóstico ${ }^{231}$.

Quanto ao tratamento instituído aos pacientes com SPCVH, chamou atenção o volume de infusão hídrica maior que $2.000 \mathrm{ml}$ nos casos fatais e menor que $2.000 \mathrm{ml}$ entre os sobreviventes (p: 0,0286). Esta observação mostra a restrição hídrica como uma importante medida terapêutica no tratamento dos pacientes com SPCVH, uma síndrome de extravasamento capilar em que os grandes volumes infundidos terminam no interstício pulmonar agravando a insuficiência respiratória ${ }^{1}$ Entretanto, alguns autores recomendam a infusão hídrica visando a aumentar o débito cardíaco e a perfusão tecidual. Porém, num estudo com 23 pacientes, realizado, na região do triângulo mineiro, os autores não encontraram relação entre sobrevida e infusão hídrica ${ }^{16}$.

O uso de corticosteróides é controverso no tratamento da SPCVH. Em altas doses inibem a agregação dos granulócitos induzida por complemento e dificultam a aderência dos neutrófilos ao endotélio da microcirculação, além de inibirem a fosfolipase e diminuírem a síntese de tromboxana e metabólitos do ácido aracdônico; portanto tem uso contra indicado na SARA além de aumentar a mortalidade de origem infecciosa ${ }^{17}$. 0 potencial efeito benéfico de doses pequenas, protegendo a membrana alvéolocapilar e prevenindo o aumento da permeabilidade vascular induzida por toxinas microbianas, pode ser considerado. Contudo, no presente estudo o uso de corticosteróide não se mostrou associado ao prognóstico dos pacientes (p: 0,7624).

0 tratamento dos pacientes com SPCVH deve ser realizado em unidade de terapia intensiva, na maioria dos casos com monitoramento cardíaco, suporte respiratório e tratamento da instabilidade hemodinâmica com aminas vasoativas e controle hídrico rigoroso.

Recomendamos a dosagem das enzimas CK e CK-MB para avaliação de acometimento da musculatura esquelética e cardíaca. Paralelamente, deve-se dosar albumina e lactato de 6 em $6 \mathrm{~h}$ como marcador de lesão tecidual grave ${ }^{1}$.

Concluímos neste estudo com base em 70 pacientes com SPCVH, a maioria da região de Ribeirão Preto (SP), a doença produziu uma letalidade de $54,3 \%$ acometendo mais indivíduos do sexo masculino com idade média de 35,8 anos; apresentou maior incidência nos meses de estiagem, de abril a setembro. Após período de incubação de 2 a 30 dias, causa febre, tosse, dispnéia, cefaléia, náuseas, vômitos, indisposição, mialgia, diarréia, fenômenos hemorrágicos e adinamia que perduram por 3 a 6 dias, com média de 4 dias, A evolução para insuficiência respiratória, hipotensão arterial e choque, na SPCVH, ocorre 24 a 48 horas após $03^{\circ}$ ou $4^{\circ}$ dia do surgimento dos sintomas. 0 hematócrito elevado e a plaquetopenia foram achados laboratoriais muito frequientes sendo sinais de suspeição diagnóstica para SPCVH. A data de maior ocorrência de óbitos é o $4^{0}$ dia após início dos sintomas. 0 exame radiológico de tórax dos pacientes mostrou velamentos bilaterais difusos, sendo intersticial no início do quadro e tendendo à alveolização difusa bilateral concomitante à insuficiência respiratória franca. A infusão hídrica maior do que $2.000 \mathrm{ml}$ e a ocorrência de hipotensão arterial, no momento da internação associaram-se à letalidade.

\section{REFERÊNCIAS}

1. Akamine N, Fernandes Jr CJ, Wey SB, Knobel E. Choque Séptico. In : Knobel E (ed) Condutas no paciente grave. Editora Atheneu, São Paulo, p. 175-210, 1994.

2. Aldala R, Galeano F, Corsiglia L. Characterization of hantavirus pulmonary syndrome patients in a critical care unit. In: Abstract book of VII International Conference on hemorragic fever renal syndrome, hantavirus pulmonary syndrome and hantaviruses, Buenos Aires, Argentina p 42, 2007.

3. Borges AA, Campos GM, Moreli ML, Souza RLM, Aquino VH, Saggioro FP, Figueiredo LTM. Hantavírus cardiopulmonary syndrome: immune response and pathogenesis. Microbes and Infection 8:2324-2330, 2006.

4. Briggler AM, Crivelli E, Pini N, Levis S, Garcia J, Diez A, Nagel J, Enria DA. Clinical spectrum of hantavirus infection in central Argentina. In: Abstract book of VII International conference on hemorragic fever renal syndrome, hantavirus pulmonary syndrome and hantaviruses, Buenos Aires, Argentina p. 47, 2007.

5. Ferres M, Vial P, Marco C, Yañez L, Godoy P, Castillo C, Hjelle B, Delgado I, Lee S, Mertz GJ. Prospective evaluation of household contacts of persons with hantavirus cardiopulmonary syndrome in Chile. Journal of Infectious Diseases, 195: 1563-1571, 2007.

6. Figueiredo LTM. Viral hemorrhagic fevers in Brazil. Revista da Sociedade Brasileira de Medicina Tropical 2:203-210, 2006.

7. Figueiredo LTM, Moreli ML, Campos GM, Sousa RL. Hantaviruses in São Paulo State, Brazil. Emerging Infectious Diseases 7:891-892, 2003.

8. Graziano KL, Tempest B. Hantavirus pulmonary syndrome: a zebra worth knowing. American Family Physician 6:1015-1020, 2002.

9. Hooper JW, Larsen T, Custer DM, Schmaljohn CS. A lethal disease model for hantavírus pulmonary syndrome. Virology 1:6-14, 2001.

10. Jonsson CB, Hooper J, Mertz G. Treatment of hantavirus pulmonary syndrome Antiviral Research 78: 162-169, 2007

11. Katz G, Tengan CH, Leite RM, Bisordi I, Kanamura CT. Epidemiologic surveillance of hantavirus cardio-pulmonary syndrome in são paulo state, Brazil, 1993-2006. In: Abstract book of VII International Conference on hemorragic fever renal syndrome, hantavirus pulmonary syndrome and hantaviruses, Buenos Aires, Argentina, p. 108, 2007.

12. Khan AS, Khabbaz RF, Armstrong LR, Holman RC, Bauer SP, Graber J, Strine T, Miller G, Reef S, Tappero J, Rollin PE, Nichol ST, Zaki SR, Bryan RT, Chapman LE, Peters CJ, Ksiazek TG. Hantavirus pulmonary syndrome: the first 100 us cases. Journal of Infectious Diseases 173:1297-1303, 1996.

13. Khan AS, Kitsutani PT, Corneli AL. Hantavirus pulmonary syndrome in the americas: the early years. Seminars in Respiratory and Critical Care Medicine 21:313-322, 2000 .

14. Kim EA, Lee KS, Primack SL, Yoon HK, Byun HS, Kim TS, Suh GY, Kwon OJ, Han J. Viral pneumonias in adults: radiologic and pathologic findings. Radiographics. p 137-149, 2002.

15. Lavocat M, Teixeira K, Wada M, Elkhoury MR Epidemiology Of Hantavirus Pulmonary Syndrome In Brazil In The Year 2005. In: Abstract book of VII 
International conference on hemorragic fever renal syndrome, hantavirus pulmonary syndrome and hantaviruses, Buenos Aires, Argentina p 109, 2007.

16. Limongi JE, Costa FC, Paula MBC, Pinto RMC, Oliveira MLA, Pajuaba Neto AA, Borges AS, Ferreira MS. Síndrome cardiopulmonar por hantavírus no triângulo mineiro e alto paranaíba, MG. 1998-2005: Aspectos clinico-epidemiológicos de 23 casos. Revista da Sociedade Brasileira de Medicina Tropical 40:72-76, 2007

17. Lorenzi Filho G, Barbas CSV, Rothman A, Amato MBP. Insuficiência respiratória aguda. In: Knobel E (org) Condutas no paciente grave. Editora Atheneu, São Paulo, p. 272-288, 1998.

18. Mc Caughey C, Hart CA. Hantaviruses. Journal of Medical Microbiology 49:587$599,2000$.

19. Marques AA, Oliveira EC, Duarte JL. Epidemiologic situation of hantavirus pulmonary syndrome, Mato Grosso, Brazil, 1999-2006 In: Abstract book of VII international conference on hemorragic fever renal syndrome, hantavirus pulmonary syndrome and hantaviruses, Buenos Aires, Argentina, p 96, 2007.

20. Moreli ML, Sousa RL, Figueiredo LTM. Detection of brazilian hantavirus by reverse transcription polymerase chain reaction amplification of $\mathrm{n}$ gene in patients with hantavirus cardiopulmonary syndrome. Memórias do Instituto Oswaldo Cruz 99:633-638, 2004.

21. Nichol ST, Arikawa J, Kawaoka Y. Emerging viral diseases. Proceedings of National Academy of Sciences 97:12411-12412, 2000.

22. Raboni SM, Probst CM, Bordignon J, Zeferino A, Santos CN. Hantaviruses in central south america: phylogenetic analysis of the s segment from hps cases in parana, Brazil. Journal of Medical Virology 76:553-562, 2005.

23. Rubinas TC, Carey RB, Kampert MC, Alkan S, Lednicky JA. Fatal hemorrhagic pneumonia concomitant with chlamydia pneumoniae and parainfluenza virus 4 infection. Archives of Pathology and Laboratory Medicine 128:640-644, 2004.

24. Rusnak JM, Huggins J, Chung K, Kim K, Bourdreau E. Intravenous ribavirin in the treatment of hemorrhagic fever renal syndrome from 1987-2005 in Korea.
In: Abstract book of VII International Conference on hemorragic fever renal syndrome, hantavirus pulmonary syndrome and hantaviruses, Buenos Aires, Argentina, p 84, 2007.

25. Saggioro FP, Rossi MA, Duarte MI, Martin CC, Alves VA, Moreli ML, Figueiredo LTM, Moreira JE, Borges AA, Neder L. Hantavirus infection induces a typical myocarditis that may be responsible for myocardial depression and shock in hantavirus pulmonary syndrome. Journal of Infection Diseases 195:1541-1549, 2007.

26. Silva MV. Brazilian Hantavirus: clinical manifestations and case-fatality rate. In: Abstract book of VII International Conference on hemorragic fever renal syndrome, hantavirus pulmonary syndrome and hantaviruses, Buenos Aires, Argentina, p 43, 2007.

27. Silva-Vergara ML, Costa Jr JC, Barata CH, Curi VG, Tiveron Jr CG, Teixeira AC. Hantavírus pulmonary syndrome in Uberaba, Minas Gerais, Brazil. Memórias do Instituto Oswaldo Cruz 97:783-787, 2002.

28. Terças ACP, Petry DMES, Kohl VA, Rosa LG, Cerqueira R, Elkhoury MR. Epidemiologica aspects of hantavírus cardiopulmonary syndrome in campo novo do parecis, Mato Grosso, Brazil. In: Abstract book of VII International Conference on hemorragic fever renal syndrome, hantavirus pulmonary syndrome and hantaviruses, Buenos Aires, Argentina, p 91, 2007.

29. Vial PA, Valdivieso F, Mertz G, Castillo C, Belmar E, Delgado I, Tapia M, Ferres M. Incubation period of hantavirus cardiopulmonary syndrome. Emerging Infection Disease 12:1271-1273, 2006.

30. Young JC, Hansen GR, Graves TK, Deasy MP, Humphreys JG, Fritz CL, Gorham KL, Khan AS, Ksiazek TG, Metzger KB, Peters CJ. The incubation period of hantavirus pulmonary syndrome. The American Journal Tropical Medicine and Hygiene 62:714-717, 2000 .

31. Zavasky DM, Hjelle B, Peterson MC, Denton RW, Reimer L. Acute infection with Sin Nombre Hantavirus without pulmonary edema. Clinical Infection Diseases 29:664-666, 1999. 